\title{
Recurrence of sigmoid colon carcinoma in the retained urethra after cystectomy: A case report and review of the literature
}

\author{
Yusuke Yagihashi, Yoshitaka Arakaki \\ Department of Urology, Okinawa Chubu Hospital.
}

\begin{abstract}
Summary Urethral recurrence arising from a primary colorectal adenocarcinoma is rare. Here, we report a case of urethral recurrence of sigmoid colon cancer, which developed after cysto-prostato-sigmoidectomy for sigmoid colon cancer invading the bladder. The patient underwent urethrectomy successfully and is currently tumor-free. Surgeons who follow patients with colorectal cancer invading the bladder should be aware of this case. The early detection of recurrence improves the chances for disease-free survival.
\end{abstract}

KEY WORDS: Fistula; Recurrence; Residual urethra; Sigmoid colon cancer.

Submitted 5 January 2015; Accepted 15 March 2015

\section{INTRODUCTION}

Primary urethral carcinoma is uncommon, and metastatic urethral carcinoma is even rarer. Here we report a case of urethral recurrence of sigmoid colon cancer, which developed after cysto-prostato-sigmoidectomy for sigmoid colon cancer invading the bladder. A complete review of the English literature on this topic was performed through PubMed search (1-11).

\section{Case report}

A 59-year-old man presented with pneumaturia and fecaluria. Cystoscopy showed an inflammatory mass on the dome of the bladder and the presence of a fistula. Colonoscopy, barium enema, and computed tomography demonstrated a 7 -cm-long sigmoid colon tumor that had invaded the bladder.

The patient underwent full-body positron emission tomography and pelvic magnetic resonance imaging (MRI) to assess for metastases. The results of these studies were negative. On laboratory examination, the carcinoembryonic antigen level was not elevated $(0.8 \mathrm{ng} / \mathrm{mL}$ [reference, $<5 \mathrm{ng} / \mathrm{mL}$ ]). Based on the diagnosis of sigmoid colon cancer with sigmoid-vesicular fistula, the patient underwent sigmoidectomy using a Hartmann's procedure combined with radical cystoprostatectomy and urinary diversion without urethrectomy.

The pathology report showed a well-differentiated adenocarcinoma with invasion into the bladder mucosa.
Nineteen lymph nodes were retrieved, and all were free of tumor. No malignancy was found in the prostate. Colonic margins were clear. Pathological diagnosis was adenocarcinoma of colonic origin, staged as pT4NOMO (stage B of Dukes classification). Post-operatively, the patient underwent 8 courses of chemotherapy with capecitabine. Forty-four months later, the patient reported a small amount of bloody discharge from the external urethral meatus. MRI showed a lesion from the urethral stump to the bulbar urethra with tumor-like qualities, but no additional metastases (Figure 1).

Urethroscopy revealed a papillary tumor that blocked the bulbar urethra completely. A urethral punch biopsy revealed a well-differentiated adenocarcinoma, which was histologically similar to the previous colon carcinoma. Based on the diagnosis of solitary urethral recurrence, a total urethrectomy was performed through the perineum.

The postoperative course was uneventful. Gross examination of the specimen demonstrated a $55 \times 30 \mathrm{~mm}$ papillary lesion inside the urethra. The pathologic examination showed well-differentiated adenocarcinoma of the urethra, consistent with the colonic primary tumor (Figure 2).

Indeed, the histologic appearance of the sigmoid colon tumor and the urethral tumor was similar. The adenocarcinoma was confined to the inner aspect of the urethra, and the surgical margins were disease free. The patient still remains recurrence-free 15 months postoperatively.

\section{Conclusion}

We report a case of urethral recurrence of sigmoid colon cancer, which developed after cysto-prostato-sigmoidectomy for sigmoid colon cancer invading the bladder.

This report is the case of recurrence of a non-urothelial malignant tumor in the residual urethra after cystoprostatectomy. Our case highlights that the residual urethra after cystoprostatectomy is a possible site for recurrence of nonurothelial malignancies.

Surgeons who follow patients with colorectal cancer invading the bladder should be aware of this case. The early detection of recurrence improves the chances for disease-free survival. 


\section{Figure 1.}

Magnetic resonance image showing a well-demarcated mass in the residual urethra.

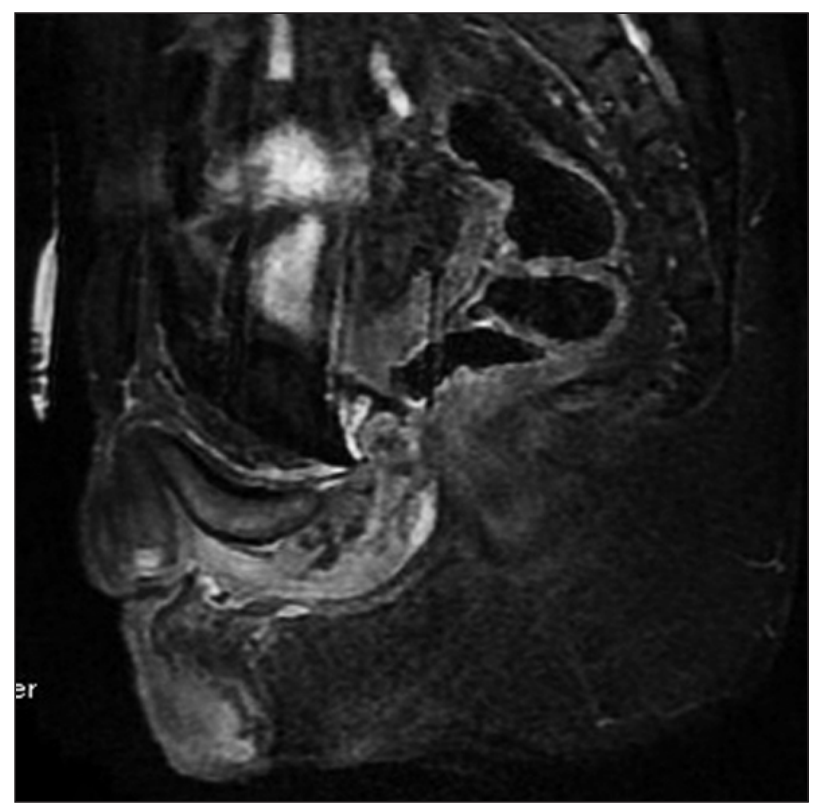

\section{Figure 2.}

Microscopic appearance of the tumor showing well-differentiated adenocarcinoma consistent with the colonic primary tumor.

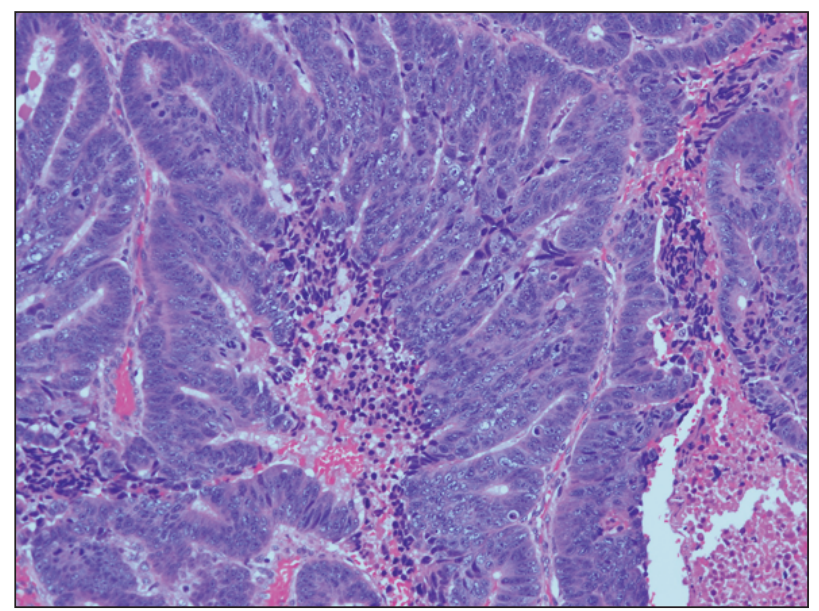

\section{Discussion is posted on www.aiua.it}

\section{References}

1. Selikowitz SM, Olsson CA. Metastatic urethral obstruction. Arch Surg. 1973; 107:906-8.

2. Okaneya T, Inoue Y, Ogawa A. Solitary urethral recurrence of sigmoid colon carcinoma. Urol Int. 1991; 47:105-7.

3. Van Thillo EL, Van Poppel H, Baert L. Urinary retention due to urethral metastases from rectal adenocarcinoma. Acta Urol Belg. 1993; 61:41-2.

4. Stragier J, Van Poppel H, Mertens V, et al. Adenocarcinoma of the rectum with a solitary metastasis to the urethra in a female. Eur $J$ Surg Oncol. 1994; 20:696-7.

5. Kupfer HW, Theunissen P, Delaere KP. Urethral metastasis from a rectal carcinoma. Acta Urol Belg. 1995; 63:31-2.

6. Yoshimura K, Isogawa Y, Yoshida H, Kawase N, Taki Y. Recurrence of sigmoid colon carcinoma in the residual urethra after cystectomy. Int J Urol. 1999; 6:479-82.

7. Chitale SV, Burgess NA, Sethia KK, et al. Management of urethral metastasis from colorectal carcinomas. ANZ J Surg. 2004; 74:925-7.

8. Chang YH, Chuang CK, Ng KF, Liao SK. Urethral metastasis from a colon carcinoma. Urology. 2007; 69:575.e1-3.

9. Noorani S, Rao AR, Callaghan PS. Urethral metastasis: an uncommon presentation of a colonic adenocarcinoma. Int Urol Nephrol. 2007; 39:837-9.

10. Martín Martín S, García Lagarto E, Egea Camacho J, Fernández Del Busto E. Urethral metastasis from a colon carcinoma 14 months alter cystoprostatectomy and sigmoidectomy. Actas Urol Esp. 2010; 34:291-3.

11. Luo HL, Tsai KL, Lin SE, Chiang PH. Outcome of urinary bladder recurrence after partial cystectomy for en bloc urinary bladder adherent colorectal cancer resection. Int J Colorectal Dis. 2013; 28:631-635.

\section{Correspondence}

Yusuke Yagihashi, MD (Corresponding Author) uroyagiyagi@yahoo.co.jp

Yoshitaka Arakaki, MD

Arakaki_yoshitaka@hosp.pref.okinawa.jp

Department of Urology, Okinawa Chubu Hospital

Okinawa, Japan 